\title{
Segmental dynamics in a blend of alkanes: Nuclear magnetic resonance experiments and molecular dynamics simulation
}

\author{
Joanne Budzien \\ Department of Chemical Engineering, University of Wisconsin-Madison, Madison, Wisconsin 53706 \\ Colleen Raphael and Mark D. Ediger \\ Department of Chemistry, University of Wisconsin-Madison, Madison, Wisconsin 53706 \\ Juan J. de Pablo \\ Department of Chemical Engineering, University of Wisconsin-Madison, Madison, Wisconsin 53706
}

(Received 7 December 2001; accepted 4 February 2002)

\begin{abstract}
The segmental dynamics of a model miscible blend, $\mathrm{C}_{24} \mathrm{H}_{50}$ and $\mathrm{C}_{6} \mathrm{D}_{14}$, were investigated as a function of temperature and composition. The segmental dynamics of the $\mathrm{C}_{24} \mathrm{H}_{50}$ component were measured with ${ }^{13} \mathrm{C}$ nuclear magnetic resonance $T_{1}$ and nuclear Overhauser effect measurements, while ${ }^{2} \mathrm{H} T_{1}$ measurements were utilized for the $\mathrm{C}_{6} \mathrm{D}_{14}$ component. Use of low molecular weight alkanes provides a monodisperse system in both components and allows differentiation of dynamics near the chain ends. From these measurements, correlation times can be calculated for the $\mathrm{C}-\mathrm{H}$ and $\mathrm{C}-\mathrm{D}$ bond reorientation as a function of component, position along the chain backbone, temperature, and composition. At $337 \mathrm{~K}$, the segmental dynamics of both molecules change by a factor of 2 to 4 across the composition range, with the central $\mathrm{C}-\mathrm{H}$ vectors of tetracosane showing a stronger composition dependence than other $\mathrm{C}-\mathrm{H}$ or $\mathrm{C}-\mathrm{D}$ vectors. Molecular simulations in the canonical and isobaric-isothermal ensembles were conducted with a united-atom force field that is known to reproduce the thermodynamic properties of pure alkanes and their mixtures with good accuracy. With a minor change to the torsion parameters, the correlation times for pure tetracosane are in good agreement with experiment. For pure hexane and its mixtures with tetracosane, the simulated dynamics are faster than experiment. () 2002 American Institute of Physics.
\end{abstract}

[DOI: $10.1063 / 1.1464538]$

\section{INTRODUCTION}

Molecular simulations have been shown to be capable of predicting the structure and thermodynamic properties of relatively complex fluids, including alkanes and polyolefins. Not as much is known about the ability of available force fields to reproduce the dynamic properties of such fluids. In this work we investigate whether a force field previously optimized for thermodynamic properties of alkanes and their mixtures can also provide an accurate description of dynamics in these systems.

Simulations of alkanes can be performed at varying levels of molecular detail. At the most detailed level, all-atom (AA) force fields consider each atom individually. At a slightly more coarse-grained level, united-atom (UA), and anisotropic-united-atom (AUA) force fields treat carbon atoms and their bonded hydrogens as a single interaction site. The UA model considers forces to act on the center of the site, while the AUA model displaces the acting forces from the center of the site. For a simple alkane, the computational demands of a simulation with a UA force field are considerably smaller than those of an all-atom calculation. That higher computational efficiency has fostered the recent development of simple force fields capable of describing the thermodynamic properties of alkanes and polyolefins. ${ }^{1-3}$ It has been postulated that UA models are not able $e^{4,5}$ to describe thermodynamic and dynamic properties of alkanes si- multaneously. This postulate, however, was proposed before the availability of more accurate UA models for thermodynamic properties. ${ }^{1,2}$

Several studies have examined the performance of UA models vis-à-vis that of $\mathrm{AA}$ models for prediction of dynamic properties. ${ }^{4,6-8}$ Simulations were performed for $n$-tridecane, $n$-tetratetracontane, and $n-\mathrm{C}_{100} \mathrm{H}_{202}$; results of UA and AA models were compared to experimental data for various dynamic properties, including $\mathrm{C}-\mathrm{H}$ bond orientation correlation times and diffusion coefficients. It was found that, in general, the UA results were worse for dynamic properties than would be expected from static-property results.

Anisotropic-united-atom models are generally perceived to offer an attractive alternative to AA models for simulations of alkanes or polyolefins. Simulations using an AUA model, for example, have been shown to provide a good description of the equation of state and the heat of vaporization of polyethylene. ${ }^{5}$ More recent calculations using the same force field indicate that good agreement with experiment can be achieved for the dipole relaxation time as a function of both temperature and pressure. ${ }^{9}$

In a recent series of papers, a group of researchers ${ }^{3,10-13}$ have compared the performance of UA and AUA models. The results have been mixed, and obscured by the use of combinations of different UA force fields, some of which are known to be inaccurate. While this body of work suggests that AUA models are superior, these conclusions could be 
partly based on inappropriate comparisons. Simulations by a different group of researchers ${ }^{14-17}$ comparing UA and AUA models did not reveal any clear advantages for dynamic properties of one over the other; each force field had strong points.

A review of the literature suggests that simulations of dynamic properties using some of the more recent and accurate UA force fields have been scarce. In this study, a series of NMR experiments were performed for systems of hexane, tetracosane, and their mixtures, to quantify fast dynamic processes as a function of temperature, composition, and field strength. Molecular dynamics simulations of the same systems were then conducted using the NERD UA force field, which has been shown to reproduce the thermodynamic properties (including phase behavior) of alkanes with good accuracy. ${ }^{1,18}$ Our results suggest that united-atom force fields are capable of describing simultaneously the equilibrium thermodynamic properties and several dynamic aspects of intermediate to long alkanes.

\section{EXPERIMENTAL METHODS AND DATA ANALYSIS}

Tetracosane $\left(\mathrm{C}_{24} \mathrm{H}_{50}\right)$ was purchased from Aldrich and used as received. Perdeuterated $n$-hexane $\left(\mathrm{C}_{6} \mathrm{D}_{14}\right)$ was purchased from Cambridge Isotope and used as received. Samples were made with the following mass fractions of $\mathrm{C}_{24} \mathrm{H}_{50}: 100 \%, 86 \%, 50 \%, 10 \%$, and 0\%. Freeze-pumpthaw cycles were used to eliminate oxygen from the samples. ${ }^{13} \mathrm{C}$ NMR $T_{1}$ and NOE experiments were performed on the $\mathrm{C}_{24} \mathrm{H}_{50}$ components of the mixtures at ${ }^{13} \mathrm{C}$ Larmor frequencies of 125.8, 75.5, and/or 62.9 MHz. ${ }^{2} \mathrm{H} T_{1}$ experiments were carried out on the $\mathrm{C}_{6} \mathrm{D}_{14}$ components of the mixtures at ${ }^{2} \mathrm{H}$ resonance frequencies of $76.9 \mathrm{and} /$ or $55.3 \mathrm{MHz}$. The temperature ranges of the experiments were chosen to ensure that the samples would be in the one-phase region for all measurements. Temperatures were calibrated against the ethylene glycol spectrum. ${ }^{19}$ For the pure $\mathrm{C}_{6} \mathrm{D}_{14}$ sample, it was necessary to use an NMR tube with a small inside diameter $(2 \mathrm{~mm})$ in order to eliminate convection. ${ }^{20}$

\section{NMR observables}

The connection between NMR observables and molecular motion is made through the orientation autocorrelation function $G(t)$ for a ${ }^{13} \mathrm{C}-\mathrm{H}$ or $\mathrm{C}-{ }^{2} \mathrm{H}$ vector. If the orientation of this vector is designated as $\mathbf{e}_{x}$, this time correlation function can be written

$$
G(t)=\frac{1}{2}\left\langle 3\left(e_{x}(0) \cdot e_{x}(t)\right)^{2}-1\right\rangle,
$$

where the brackets indicate an average over all possible starting times $t=0$ and over all nuclei with the same resonance frequency. The spectral density, $J(\omega)$, is the Fourier transform of $G(t)$,

$$
J(\omega)=\frac{1}{2} \int_{-\infty}^{\infty} e^{i \omega t} G(t) d t .
$$

The correlation time $\tau_{c}$ is defined as the time integral of $G(t)$,

$$
\tau_{c}=\int_{0}^{\infty} G(t) d t .
$$

\section{Deuterated hexane dynamics}

Because ${ }^{2} \mathrm{H}$ nuclei relax via the quadrupolar mechanism, the relationship between $T_{1}$ and $J(\omega)$ is

$$
\frac{1}{T_{1}}=\frac{3}{10} \pi^{2}\left(\frac{e^{2} q Q}{h}\right)^{2}\left[J\left(\omega_{D}\right)+4 J\left(2 \omega_{D}\right)\right] .
$$

All the ${ }^{2} \mathrm{H}$ experiments reported here are in the extreme narrowing limit. In this case, the spectral density function is equal to its zero frequency limit and there exists an inverse relationship between the correlation time $\tau_{c}$ and $T_{1}$ :

$$
\frac{1}{T_{1}}=\frac{3}{2} \pi^{2}\left(\frac{e^{2} q Q}{h}\right)^{2} \tau_{c} .
$$

For $\mathrm{C}_{6} \mathrm{D}_{14}$, the quadrupole coupling constant $\left(e^{2} q Q / h\right)^{2}$ is equal to $168 \mathrm{KHz}$ for deuterons attached to the $\alpha$ carbon (methyl groups) and $172 \mathrm{KHz}$ for the $\beta$ and $\gamma$ deuterons. The $\alpha$ deuterons are detectable as one peak in the spectrum while the $\beta$ and $\gamma$ deuterons are detectable as a second peak.

\section{Tetracosane dynamics}

Five peaks are resolved in the ${ }^{13} \mathrm{C}$ NMR spectrum of $\mathrm{C}_{24} \mathrm{H}_{50}$, corresponding to the four carbons at each end of the chain $(\alpha=$ methyl, $\beta, \gamma, \delta)$ and the 16 carbons in the center of the chain. Hence the dynamics of five types of $\mathrm{C}-\mathrm{H}$ vectors can be investigated. Dipole-dipole (DD) interactions are the main relaxation mechanism for these experiments. In some cases, spin rotation (SR) also plays a role. The observed ${ }^{13} \mathrm{C}$ relaxation rate can be expressed

$$
\frac{1}{T_{1}}=\frac{1}{T_{1}^{\mathrm{DD}}}+\frac{1}{T_{1}^{\mathrm{SR}}}
$$

The separation of the measured $T_{1}$ into its two components $\left(T_{1}^{\mathrm{DD}}\right.$ and $\left.T_{1}^{\mathrm{SR}}\right)$ can be accomplished using the experimentally measured nuclear Overhauser effect (NOE) values. The NOE value associated with dipole-dipole interaction $\mathrm{NOE}^{\mathrm{DD}}$ is bounded below by the observed NOE and above by 2.99 . NOE ${ }^{\mathrm{DD}}$ should increase or remain constant as the temperature increases, as the concentration of $\mathrm{C}_{24} \mathrm{H}_{50}$ decreases, as the resonance frequency decreases, and as the end of the chain is approached. Most of our data showed these trends; in these cases, we set $\mathrm{NOE}^{\mathrm{DD}}$ equal to the observed NOE and $T_{1}^{\mathrm{DD}}$ equal to the observed $T_{1}$. When the data did not show these trends, $\mathrm{NOE}^{\mathrm{DD}}$ was estimated using the above expectations, and the $T_{1}^{\mathrm{DD}}$ was calculated from

$$
T_{1}^{\mathrm{DD}}=\left(\frac{\left[\mathrm{NOE}^{\mathrm{DD}}-1\right]}{[\mathrm{NOE}-1]}\right) * T_{1} .
$$

This $T_{1}$ correction was usually less than $5 \%$ and always less than $17 \%$, except for the $\alpha$ and $\beta$ carbons. The expected uncertainty in this procedure is $\leqslant 10 \%$ in $\tau_{c}$, except for the $\alpha$ carbons where the error may be somewhat larger.

When $\mathrm{NOE}^{\mathrm{DD}}$ is near its maximum value of 2.99 , extreme narrowing conditions apply and the correlation time can be calculated directly from $T_{1}$,

$$
\tau_{c}=\frac{1}{10 K n T_{1}^{\mathrm{DD}}} .
$$


TABLE I. NERD potential parameters.

Bond stretching potential

$\frac{V(r)}{k_{B}}=\frac{K_{r}}{2}\left(r-b_{\mathrm{eq}}\right)^{2}$

$K_{r}=96500 \mathrm{~K} / \AA^{2}, b_{\mathrm{eq}}=1.54 \AA$

Bond bending potential

$\frac{V(\theta)}{k_{B}}=\frac{K_{\theta}}{2}\left(\theta-\theta_{\mathrm{eq}}\right)^{2}$

$K_{\theta}=62500 \mathrm{~K} / \mathrm{rad}^{2}, \theta_{\mathrm{eq}}=114.0^{\circ}$

Torsion potential

$\frac{V(\phi)}{k_{B}}=V_{0}+V_{1}(1+\cos \phi)+V_{2}(1-\cos 2 \phi)+V_{3}(1+\cos 3 \phi)$

$V_{0}=0 \mathrm{~K}, V_{1}=355.04 \mathrm{~K}$

$V_{2}=-68.19 \mathrm{~K}, V_{3}=701.32 \mathrm{~K}$

Nonbonded potential

$\frac{V(r)}{k_{B}}=4 \epsilon\left[\left(\frac{\sigma}{r}\right)^{12}-\left(\frac{\sigma}{r}\right)^{6}\right]$

$\sigma_{\mathrm{CH}_{2}}=3.93 \AA, \epsilon_{\mathrm{CH}_{2}}=45.8 \mathrm{~K}$

$\sigma_{\mathrm{CH}_{3}}=3.91 \AA, \epsilon_{\mathrm{CH}_{3}}=104.0 \mathrm{~K}$

Here the constant $K$ is taken to be $2.29 \times 10^{9} \mathrm{~s}^{-2},{ }^{21}$ and $n$ is the number of bonded protons. Outside the extreme narrowing regime, $T_{1}^{\mathrm{DD}}$ and $\mathrm{NOE}^{\mathrm{DD}}$ depend upon the spectral density function as follows:

$$
\begin{aligned}
& \frac{1}{T_{1}^{\mathrm{DD}}}=K n\left[J\left(\omega_{\mathrm{H}}-\omega_{\mathrm{C}}\right)+3 J\left(\omega_{\mathrm{C}}\right)+6 J\left(\omega_{\mathrm{H}}+\omega_{\mathrm{C}}\right)\right], \\
& \mathrm{NOE}^{\mathrm{DD}}=1+\frac{\gamma_{\mathrm{H}}}{\gamma_{\mathrm{C}}}\left[\frac{6 J\left(\omega_{\mathrm{H}}+\omega_{\mathrm{C}}\right)-J\left(\omega_{\mathrm{H}}-\omega_{\mathrm{C}}\right)}{J\left(\omega_{\mathrm{H}}-\omega_{\mathrm{C}}\right)+3 J\left(\omega_{\mathrm{C}}\right)+6 J\left(\omega_{\mathrm{H}}+\omega_{\mathrm{C}}\right)}\right],
\end{aligned}
$$

where $\gamma_{\mathrm{H}}$ and $\gamma_{\mathrm{C}}$ are the gyromagnetic ratios for carbon and hydrogen, and $\omega_{\mathrm{H}}$ and $\omega_{\mathrm{C}}$ are their respective resonance frequencies. Based on previous experiments on oligomeric polyethylene melts, we have estimated the shape of $J(\omega)$ for $\mathrm{C}_{24} \mathrm{H}_{50}$ and used this to estimate the correlation time when extreme narrowing conditions do not apply. This procedure is described in the Appendix. We estimate that this calculation of correlation times introduces a systematic error of no more than $10 \%$.

\section{THEORY AND SIMULATION METHODS}

Both NVT and NPT molecular dynamics simulations were performed in this work. The NVT simulations used a Nosé-Hoover thermostat ${ }^{22,23}$ with a temperature coupling parameter of $0.2 \mathrm{ps}$. The NPT simulations were performed using the method of Berendsen et al. ${ }^{24}$ with a temperature coupling parameter of 8 ps and a pressure coupling parameter of 16 ps. The time step was 0.814 fs. A velocity Verlet algorithm was used to integrate the equations of motion for both types of simulations. For all the systems, at least $40 \mathrm{ps}$ of equilibration time were allowed before data collection began.

The NERD potential ${ }^{18}$ used in this work is shown in Table I. The torsion potential was systematically changed as described below. A cutoff radius of $13 \AA$ was used for the
Lennard-Jones interactions, and tail corrections were applied. System sizes were set so that there were 2400 to 5000 interaction sites in the simulation box.

The simulated quantity of interest to this work is the orientation autocorrelation function, $G(t)$, defined in Eq. (1) for the $\mathrm{C}-\mathrm{H}$ bond vector. Given that the UA model used here does not consider hydrogen atoms explicitly, it is necessary to develop alternative methods of interpreting our data in terms of implicit $\mathrm{C}-\mathrm{H}$ bonds. The position of the interaction site is taken as that of the carbon. The hydrogens are assumed to be located in the plane formed by the bisector vector and the out-of-plane vector of the three backbone carbons (the carbon of interest and its two nearest neighbors), with a $\mathrm{H}-\mathrm{C}-\mathrm{H}$ angle of $109.5^{\circ} .^{25}$ The motion of the hydrogens in the terminal methyl groups ( $\alpha$ carbon) cannot be inferred from a UA model.

In view of the fast initial decay of the correlation functions, and given the long-time tails encountered in some systems, it was practical to consider multiple time-step sizes to average $G(t)$ and then splice the results. For the shortest times, multiple $G(t)$ 's were calculated using 100 time-step increments and averaged together; 1000 time-step increments were used to calculate $G(t)$ 's over the entire simulation time. The finer grid was used at short times (generally less than 10 ps) until noise or deviations from the coarser grid were evident.

To simplify the calculations of $\tau_{c}$ [from Eq. (3)], the $G(t)$ function is fit to a modified Kohlrasch-Williams-Watts (mKWW) equation ${ }^{26}$

$$
G(t)=A e^{-\left(t / \tau_{\mathrm{KWW}}\right)^{\beta}}+(1-A) e^{-t / \tau_{0}},
$$

where $A, \beta, \tau_{\mathrm{KWw}}$, and $\tau_{0}$ are adjustable parameters. The form of this equation is empirical, and has been used in previous studies of $G(t) .{ }^{27-29}$ The parameters must satisfy the constraints $0 \leqslant \beta \leqslant 1,0 \leqslant A \leqslant 1, \tau_{\mathrm{KWw}}>0$, and $\tau_{0}>0$. The correlation time is then calculated by integrating Eq. (11) over time:

$$
\tau_{c}=\frac{A \tau_{\mathrm{KWW}}}{\beta} \Gamma\left(\frac{1}{\beta}\right)+(1-A) \tau_{0},
$$

where $\Gamma$ is the gamma function.

\section{EXPERIMENTAL RESULTS}

Tables II-VI present the NMR measurements on $\mathrm{C}_{24} \mathrm{H}_{90} / \mathrm{C}_{6} \mathrm{D}_{14}$ mixtures, and the correlation times which we have calculated from these measurements. Figures 1 and 2 show the temperature dependence of the correlation times for $\mathrm{C}-\mathrm{H}$ and $\mathrm{C}-\mathrm{D}$ vectors in neat tetracosane and in neat hexane, respectively (the simulation results shown in these figures will be discussed below). In Fig. 1, correlation times are shown for the five different types of $\mathrm{C}-\mathrm{H}$ vectors resolvable in these experiments. Over the temperature range from 328 to $337 \mathrm{~K}$, all $\mathrm{C}-\mathrm{H}$ vectors show the same change in correlation times (21-28\%) within experimental error. Based on the Debye-Stokes-Einstein equation, one might expect the correlation time changes to have the same temperature dependence as the viscosity $\eta$ divided by the absolute temperature. In agreement with this expectation, $\eta / T$ for pure tetracosane 
TABLE II. $\mathrm{C}_{24} \mathrm{H}_{50} T_{1}(s)$ ( $T_{1}^{\text {DD }}$ values shown in parentheses if different).

\begin{tabular}{|c|c|c|c|c|c|c|}
\hline $\mathrm{C}_{24} \mathrm{H}_{50}$ & $T(\mathrm{~K})$ & $\alpha T_{1}$ & $\beta T_{1}$ & $\gamma T_{1}$ & $\delta T_{1}$ & Center $T_{1}$ \\
\hline \multirow[t]{5}{*}{$100 \%$} & $337^{\mathrm{a}}$ & $6.5 \pm 0.3$ & $4.3 \pm 0.2$ & $3.0 \pm 0.1$ & $2.2 \pm 0.1$ & $1.22 \pm 0.05$ \\
\hline & $329^{a}$ & $5.56 \pm 0.06$ & $3.48 \pm 0.03$ & $2.45 \pm 0.02$ & $1.79 \pm 0.02$ & $1.02 \pm 0.01$ \\
\hline & $337^{\mathrm{b}}$ & $6.5 \pm 0.1$ & $4.3 \pm 0.1$ & $3.1 \pm 0.1$ & $2.3 \pm 0.1$ & $1.32 \pm 0.02$ \\
\hline & & (7.0) & (4.5) & (3.2) & & \\
\hline & $327^{\mathrm{b}}$ & $5.7 \pm 0.2$ & $3.79 \pm 0.06$ & $2.74 \pm 0.06$ & $2.04 \pm 0.05$ & $1.21 \pm 0.04$ \\
\hline \multirow[t]{3}{*}{$50 \%$} & $337^{\mathrm{b}}$ & $\begin{array}{l}7.5 \pm 0.4 \\
(8.0)\end{array}$ & $\begin{array}{l}6.3 \pm 0.3 \\
(6.6)\end{array}$ & $\begin{array}{l}4.8 \pm 0.3 \\
(4.9)\end{array}$ & $\begin{array}{l}3.7 \pm 0.2 \\
(3.8)\end{array}$ & $2.06 \pm 0.08$ \\
\hline & $327^{\mathrm{b}}$ & $\begin{array}{l}\quad 6.8 \pm 0.2 \\
(7.3)\end{array}$ & $\begin{array}{l}5.5 \pm 0.2 \\
(5.6)\end{array}$ & $4.2 \pm 0.1$ & $3.2 \pm 0.1$ & $1.84 \pm 0.07$ \\
\hline & $317^{\mathrm{b}}$ & $6.0 \pm 0.1$ & $4.9 \pm 0.1$ & $3.7 \pm 0.1$ & $2.9 \pm 0.2$ & $1.7 \pm 0.2$ \\
\hline \multirow[t]{2}{*}{$10 \%$} & $337^{\mathrm{a}}$ & $\begin{array}{l}9.1 \pm 2.0 \\
(14.3)\end{array}$ & $\begin{array}{l}9.1 \pm 1.2 \\
(10.8)\end{array}$ & $\begin{array}{l}8.5 \pm 0.8 \\
(9.2)\end{array}$ & $\begin{array}{l}\quad 6.7 \pm 1.3 \\
(7.3)\end{array}$ & $\begin{array}{l}3.2 \pm 0.1 \\
(3.7)\end{array}$ \\
\hline & $307^{\mathrm{a}}$ & $\begin{array}{l}6.8 \pm 0.2 \\
(7.5)\end{array}$ & $\begin{array}{l}6.7 \pm 0.2 \\
(7.1)\end{array}$ & $5.5 \pm 0.3$ & $4.2 \pm 0.2$ & $2.48 \pm 0.02$ \\
\hline
\end{tabular}

${ }^{\mathrm{a}} 75.5 \mathrm{MHz}$.

${ }^{\mathrm{b}} 125.8 \mathrm{MHz}$.

TABLE III. $\mathrm{C}_{24} \mathrm{H}_{50}$ NOE values ( $\mathrm{NOE}^{\mathrm{DD}}$ shown in parentheses if different). Errors are \pm 0.05 .

\begin{tabular}{|c|c|c|c|c|c|c|}
\hline $\mathrm{C}_{24} \mathrm{H}_{50}$ & $T(\mathrm{~K})$ & $\alpha$ & $\beta$ & $\gamma$ & $\delta$ & Center \\
\hline \multirow[t]{4}{*}{$100 \%$} & $337^{\mathrm{a}}$ & $2.93(2.95)$ & 2.93 & 2.89 & 2.91 & 2.80 \\
\hline & $329^{\mathrm{a}}$ & 2.94 & 2.91 & 2.86 & 2.84 & 2.72 \\
\hline & $337^{\mathrm{b}}$ & $2.79(2.94)$ & $2.83(2.92)$ & $2.78(2.86)$ & 2.76 & 2.60 \\
\hline & $327^{\mathrm{b}}$ & 2.96 & 2.79 & 2.80 & 2.70 & 2.51 \\
\hline \multirow[t]{3}{*}{$50 \%$} & $337^{\mathrm{b}}$ & $2.85(2.98)$ & $2.85(2.95)$ & $2.85(2.90)$ & $2.86(2.88)$ & 2.84 \\
\hline & $327^{\mathrm{b}}$ & $2.85(2.98)$ & $2.90(2.93)$ & 2.91 & 2.85 & 2.81 \\
\hline & $317^{\mathrm{b}}$ & 2.95 & 2.93 & 2.90 & 2.88 & 2.79 \\
\hline \multirow[t]{2}{*}{$10 \%$} & $337^{\mathrm{a}}$ & $2.27(2.99)$ & $2.67(2.99)$ & $2.85(2.99)$ & $2.77(2.92)$ & $2.63(2.89)$ \\
\hline & $307^{\mathrm{a}}$ & $2.80(2.99)$ & $2.87(2.99)$ & $2.96(2.99)$ & 2.94 & 2.77 \\
\hline
\end{tabular}

${ }^{\mathrm{a}} 75.5 \mathrm{MHz}$.

${ }^{\mathrm{b}} 125.8 \mathrm{MHz}$.

TABLE IV. $\mathrm{C}_{24} \mathrm{H}_{50}$ correlation times (ps).

\begin{tabular}{|c|c|c|c|c|c|c|}
\hline $\mathrm{C}_{24} \mathrm{H}_{50}$ & $T(\mathrm{~K})$ & $\alpha$ & $\beta$ & $\gamma$ & $\delta$ & Center \\
\hline \multirow[t]{2}{*}{$100 \%$} & 337 & $2.2 \pm 0.2$ & $5.3 \pm 0.3$ & $7.5 \pm 0.4$ & $11 \pm 1$ & $21 \pm 2$ \\
\hline & 328 & $2.7 \pm 0.4$ & $6.6 \pm 0.2$ & $9.4 \pm 0.4$ & $13.3 \pm 0.6$ & $27 \pm 2$ \\
\hline \multirow[t]{3}{*}{$50 \%$} & 337 & $1.8 \pm 0.1$ & $3.3 \pm 0.2$ & $4.8 \pm 0.3$ & $6.2 \pm 0.3$ & $11.7 \pm 0.5$ \\
\hline & 327 & $2.0 \pm 0.1$ & $3.9 \pm 0.2$ & $5.5 \pm 0.2$ & $7.4 \pm 0.3$ & $13.4 \pm 0.5$ \\
\hline & 317 & $2.4 \pm 0.1$ & $4.5 \pm 0.1$ & $6.3 \pm 0.2$ & $8.2 \pm 0.5$ & $15 \pm 2$ \\
\hline \multirow[t]{2}{*}{$10 \%$} & 337 & $1.0 \pm 0.3$ & $2.0 \pm 0.3$ & $2.4 \pm 0.3$ & $3.1 \pm 0.6$ & $6.4 \pm 0.3$ \\
\hline & 307 & $1.9 \pm 0.1$ & $3.1 \pm 0.3$ & $4.1 \pm 0.4$ & $5.5 \pm 0.3$ & $10.2 \pm 0.2$ \\
\hline
\end{tabular}

TABLE V. $\mathrm{C}_{6} \mathrm{D}_{14} T_{1}$ values and correlation times.

\begin{tabular}{cccccc}
\hline \hline $\mathrm{C}_{6} \mathrm{D}_{14}$ & $T(\mathrm{~K})$ & $\beta \gamma T_{1}(\mathrm{~s})$ & $\alpha T_{1}(\mathrm{~s})$ & $\beta \gamma \tau_{c}(\mathrm{ps})$ & $\alpha \tau_{c}(\mathrm{ps})$ \\
\hline $100 \%$ & $337^{\mathrm{a}}$ & $2.41 \pm 0.01$ & $2.66 \pm 0.02$ & $0.95 \pm 0.01$ & $1.03 \pm 0.01$ \\
& $327^{\mathrm{a}}$ & $2.21 \pm 0.03$ & $2.38 \pm 0.01$ & $1.13 \pm 0.01$ & $1.01 \pm 0.01$ \\
& $317^{\mathrm{a}}$ & $2.02 \pm 0.02$ & $2.18 \pm 0.04$ & 1.01 \\
& $307^{\mathrm{a}}$ & $1.80 \pm 0.05$ & $1.93 \pm 0.06$ & $1.74 \pm 0.02$ & $1.03 \pm 0.02$ \\
$50 \%$ & $337^{\mathrm{b}}$ & $1.31 \pm 0.02$ & $1.77 \pm 0.01$ & $2.17 \pm 0.02$ & $1.35 \pm 0.01$ \\
& $327^{\mathrm{b}}$ & $1.20 \pm 0.01$ & $1.60 \pm 0.01$ & 2.00 .04 & $1.49 \pm 0.01$ \\
$14 \%$ & $317^{\mathrm{b}}$ & $1.05 \pm 0.01$ & $1.43 \pm 0.01$ & $3.0 \pm 0.1$ & $1.68 \pm 0.02$ \\
& $337^{\mathrm{b}}$ & $0.91 \pm 0.03$ & $1.44 \pm 0.03$ & $1.26 \pm 0.10$ & $1.90 \pm 0.0 .14$ \\
\hline \hline
\end{tabular}

a55.3 and $76.4 \mathrm{MHz}$.

b55.3 MHz.

Downloaded 05 Mar 2007 to 128.104.198.71. Redistribution subject to AIP license or copyright, see http://jcp.aip.org/jcp/copyright.jsp 
TABLE VI. $T_{1}$, NOE, and correlation times for center carbons of neat $\mathrm{C}_{24} \mathrm{H}_{50}(T \geqslant 341 \mathrm{~K})$.

\begin{tabular}{cccr}
\hline \hline$T(\mathrm{~K})$ & $T_{1}^{\mathrm{a}}(\mathrm{s})$ & $\mathrm{NOE}^{\mathrm{a}}( \pm 0.05)$ & $\tau_{c}(\mathrm{ps})$ \\
\hline 371 & $2.26 \pm 0.05$ & 2.98 & $9.8 \pm 0.5$ \\
365 & $2.08 \pm 0.04$ & 2.91 & $11.0 \pm 0.6$ \\
360 & $1.90 \pm 0.04$ & 2.88 & $12.4 \pm 0.6$ \\
354 & $1.69 \pm 0.03$ & 2.95 & $13.3 \pm 0.7$ \\
347 & $1.53 \pm 0.03$ & 2.91 & $15.1 \pm 0.8$ \\
341 & $1.33 \pm 0.03$ & 2.90 & $17.4 \pm 0.9$ \\
\hline \hline
\end{tabular}

${ }^{\mathrm{a}} 62.9 \mathrm{MHz}$.

changes by $24 \%$ over this temperature range. ${ }^{30}$ Figure 2 shows the temperature dependence of the two different types of resolved $\mathrm{C}-\mathrm{D}$ vectors in neat per-deuterated hexane. The $\alpha$ and $\beta \gamma \mathrm{C}-\mathrm{D}$ vectors both show about a $36 \%$ change in correlation times over the temperature range from 307 to 337 $\mathrm{K}$. Over this temperature range, $\eta / T$ for undeuterated hexane changes by $40 \%,{ }^{31}$ again consistent with the changes in correlation times within experimental error.

Figures 1 and 2 show a strong similarity between macroscopic and microscopic measures of liquid dynamics for tetracosane and hexane as neat components. Interestingly, this correlation works for the central $\mathrm{C}-\mathrm{H}$ vectors and those close to the chain ends, including the methyl groups. The former are partially influenced by the longest relaxation times of the alkane molecules, while the reorientation of the methyl groups is a considerably more localized motion.

The connection between microscopic dynamics and viscosity is much more complicated in the mixtures of tetracosane and hexane. Figure 3 shows the dynamics of all types of $\mathrm{C}-\mathrm{H}$ and $\mathrm{C}-\mathrm{D}$ vectors as a function of composition at 337 $\mathrm{K}$. For all the $\mathrm{C}-\mathrm{H}$ and $\mathrm{C}-\mathrm{D}$ vectors, changes in composition cause changes in correlation times by factors of 1.8 to 3.6. In contrast, the viscosity increases by a factor of 18 as the composition is changed from pure hexane to pure tetracosane. This difference between macroscopic and microscopic measures of dynamics would be qualitatively anticipated for this case. The viscosity depends most strongly on the longest relaxation times of the molecules, while the $\mathrm{C}-\mathrm{H}$

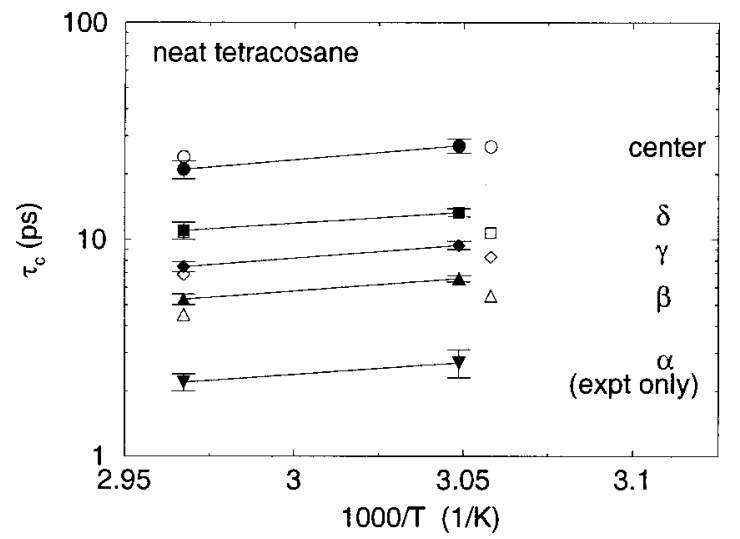

FIG. 1. Correlation times for the different $\mathrm{C}-\mathrm{H}$ vectors in neat tetracosane as a function of temperature. Solid symbols are experimental points, open symbols are simulated points using the $15 \%$ higher torsion potential.

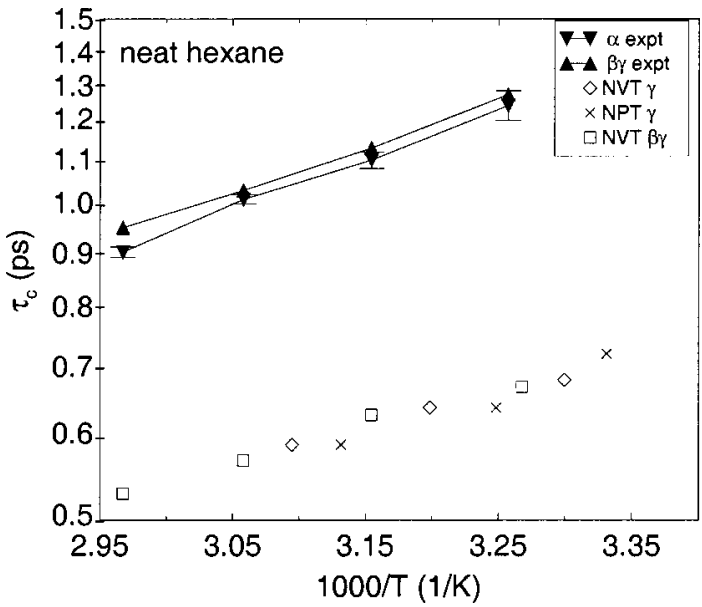

FIG. 2. Correlation times for $\mathrm{C}-\mathrm{D}$ vectors in neat hexane as a function of temperature. Note that NPT simulated points are for $\gamma$ carbon C-D vectors only.

and $\mathrm{C}-\mathrm{D}$ vector correlation times are mainly sensitive to conformational relaxation times which are not as strongly affected by composition changes.

Two further observations for these mixtures do not have simple explanations. Close scrutiny of Fig. 3 shows that the more central $\mathrm{C}-\mathrm{H}$ vectors of tetracosane (center, $\delta$, and $\gamma$ ) show a stronger composition dependence (dynamics change by about a factor of 3.3) than the other $\mathrm{C}-\mathrm{H}$ or $\mathrm{C}-\mathrm{D}$ vectors (dynamics change by about a factor of 2.4). For the 50/50 mixture, over the temperature range of 317 to $337 \mathrm{~K}$, the $\mathrm{C}-\mathrm{H}$ vectors in tetracosane change shift dynamics by $28-$ $36 \%$, while the $\mathrm{C}-\mathrm{D}$ vectors change by $25 \%$. While this difference is just barely significant, given the error bars, it is consistent with a trend seen in some miscible polymer blends, i.e., that each component retains its own temperature dependence in the blend. ${ }^{32}$

\section{SIMULATION RESULTS}

\section{Tetracosane}

Several simulations were run for pure tetracosane systems in the temperature range $324-371 \mathrm{~K}$. Sample $\mathrm{C}-\mathrm{H}$ vec-

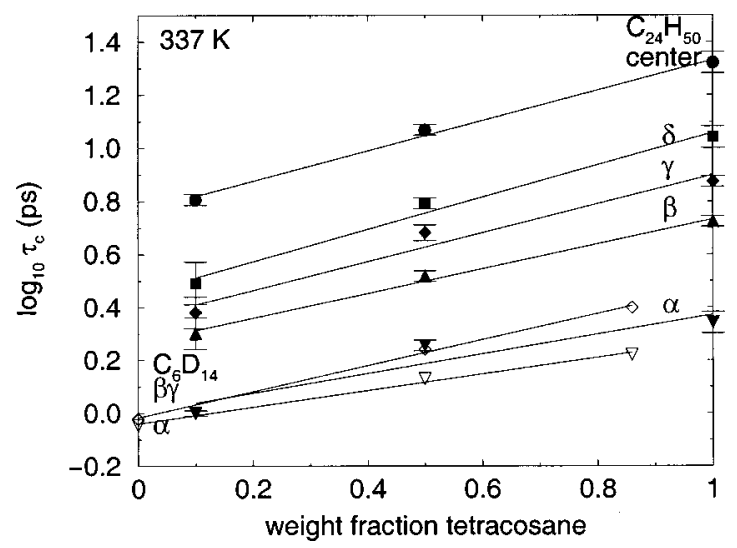

FIG. 3. Experimental correlation times for mixtures of hexane and tetracosane as a function of composition at $337 \mathrm{~K}$. 


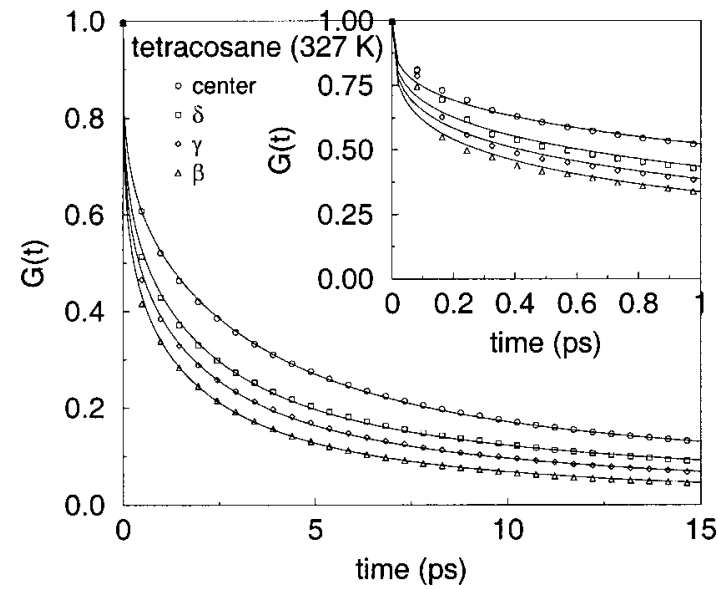

FIG. 4. Representative orientation autocorrelation functions for $\mathrm{C}-\mathrm{H}$ vectors in tetracosane at $327 \mathrm{~K}$, obtained with standard NERD torsion. Lines are mKWW fits.

tor orientation autocorrelation functions for the four sets of carbons accessible from simulation $(\beta, \gamma, \delta$, and center) are shown in Fig. 4. A fast initial decay is seen in the first $0.3 \mathrm{ps}$ of the correlation function, followed by a much more gradual process. Figure 5 shows an extended temperature range for the center-carbons correlation times and compares two sets of simulated results with the corresponding experimental values. It can be seen that the standard NERD potential simu-

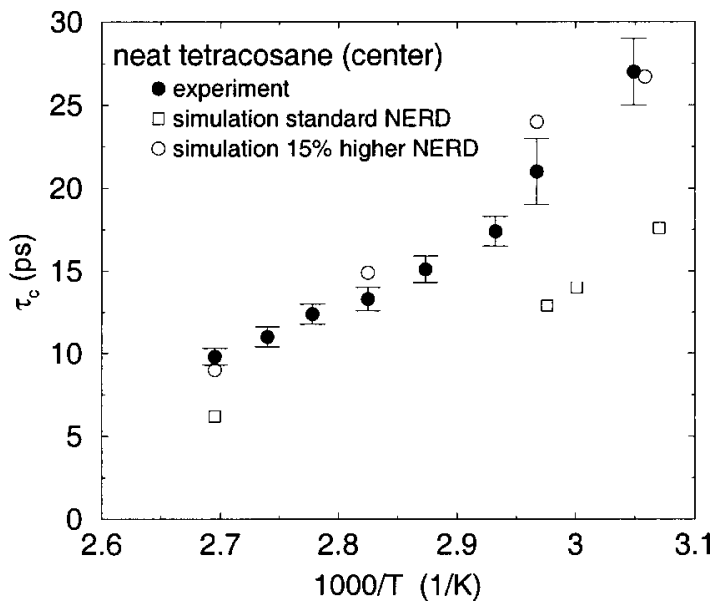

FIG. 5. Comparison of simulated and experimental correlation times for the center $\mathrm{C}-\mathrm{H}$ vectors of tetracosane as a function of temperature. Solid symbols are experimental points, open symbols are simulated points.

lated results are faster than the experimental results by approximately $50 \%$. Results by Smith et al. ${ }^{33}$ on polybutadiene show that changes in the torsional potential have a very significant effect on $\mathrm{C}-\mathrm{H}$ vector reorientation in longer molecules. They report that raising the barrier between conformational states by only $0.4 \mathrm{kcal} / \mathrm{mol}$, slowed $\mathrm{C}-\mathrm{H}$ vector relaxation times by a factor of 2 . As a rough means to im-

TABLE VII. Simulation results for neat tetracosane.

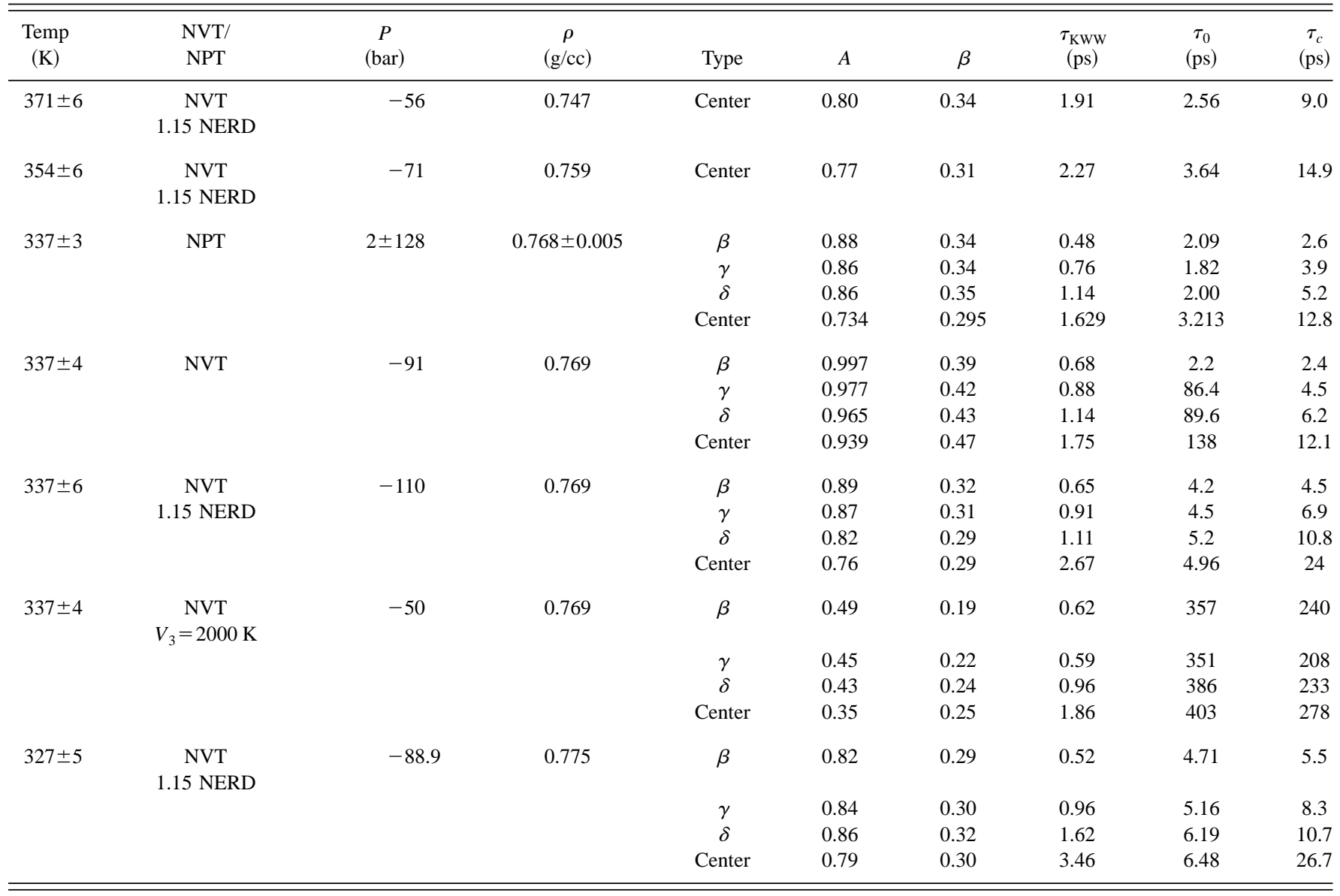




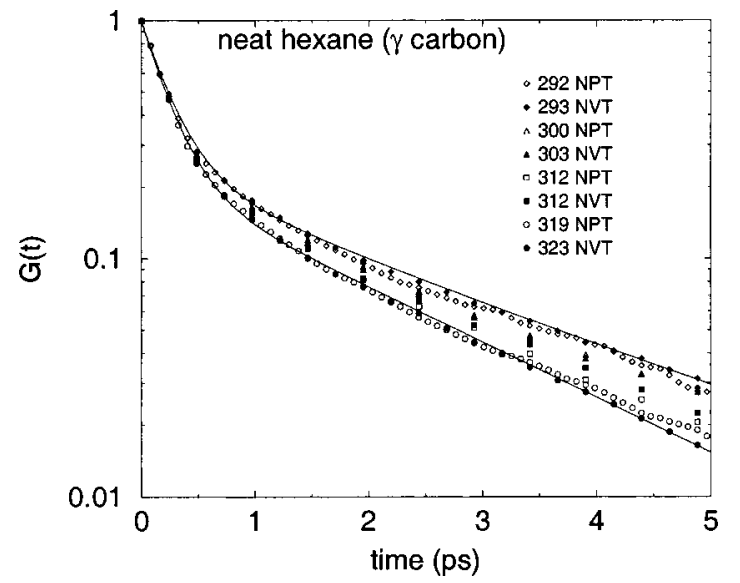

FIG. 6. Comparison of $\gamma$ carbon $\mathrm{C}-\mathrm{H}$ vector orientation autocorrelation functions from standard NERD force field NVT and NPT simulations of neat hexane. Solid symbols correspond to NVT results, open symbols correspond to NPT results. Lines are NVT mKWW fits.

prove agreement between simulation and experiment, the torsion potential in this work was modified by multiplying the NERD torsion potential parameters by 1.15 (raising the barriers by about $15 \%$ for an increase of $0.45 \mathrm{kcal} / \mathrm{mol}$ ) and simulations were run using this potential. The inset in Fig. 7 shows the modified torsion and the standard torsion. Although we have not verified this point, it is expected that, since equilibrium properties depend on the minima in the torsion potential, this change will not influence the thermodynamic properties to a significant extent. This modification resulted in good agreement between simulation and experiment, as can be seen in Figs. 1 and 5. Table VII gives the parameters for the fit to the simulation data for pure tetracosane using Eq. (11), the modified torsion potential, and compares the results of the torsion modification at $337 \mathrm{~K}$.

\section{Hexane}

Hexane simulations were run at temperatures between $285 \mathrm{~K}$ and $322 \mathrm{~K}$ with both NPT and NVT dynamics. Figure 6 compares the NVT and NPT results for the $\mathrm{C}-\mathrm{H}$ vector

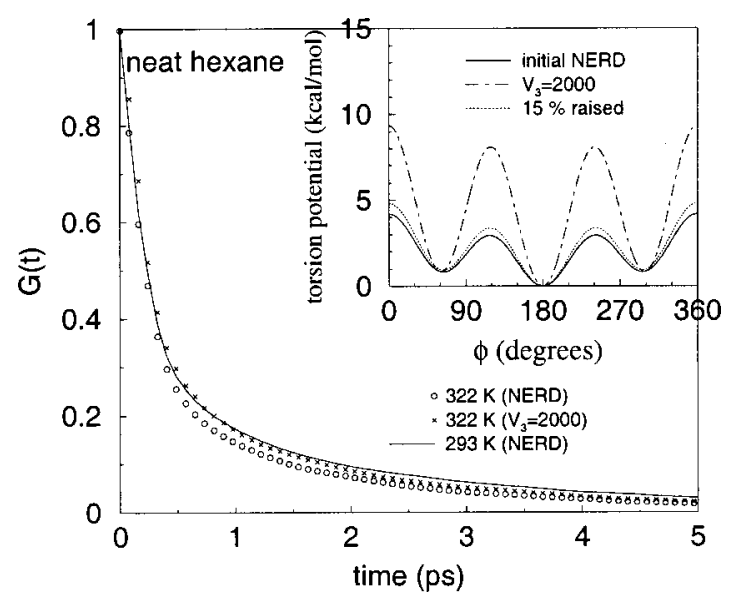

FIG. 7. Effect of changing the torsional potential on the orientation autocorrelation function for the $\gamma$ carbon $\mathrm{C}-\mathrm{H}$ vector of neat hexane. The energies of the torsional potentials are compared in the inset.

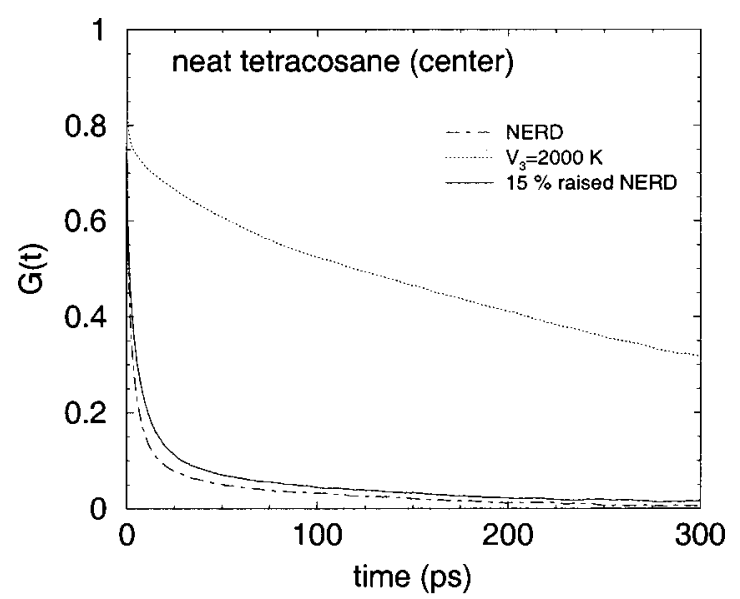

FIG. 8. Simulated center carbon tetracosane $\mathrm{C}-\mathrm{H}$ vector dynamics with changing torsion potential.

autocorrelation function $G(t)$. It can be seen that the NPT and NVT simulations give similar results.

Figure 2 compares the simulated and experimental correlation times. The simulated correlation times are about half as long as the corresponding experimental values, consistent with observations for tetracosane. As with tetracosane, we increased the torsion energy barriers in an attempt to improve agreement with experiment. The trans-gauche and cis barriers were increased by roughly $5 \mathrm{kcal} / \mathrm{mol}$. This was accomplished by changing $V_{3}$ to $2000 \mathrm{~K}$ for hexane. The inset in Fig. 7 shows both the original and the modified torsion potentials. An NPT simulation of pure hexane at $322 \mathrm{~K}, 0.9$ bar was run with this modified potential.

The resulting $G(t)$ for the $\gamma$ carbon is compared to the standard torsion potential results in Fig. 7, at both $322 \mathrm{~K}$ and $293 \mathrm{~K}$. The change in correlation time from $0.60 \mathrm{ps}$ to 0.69 ps corresponds to only a $15 \%$ increase at $322 \mathrm{~K}$; this result is still well below the experimental time of about $1.08 \mathrm{ps}$. In contrast, using this greatly increased torsion potential for tetracosane slows the dynamics by an enormous amount, as is shown in Fig. 8.

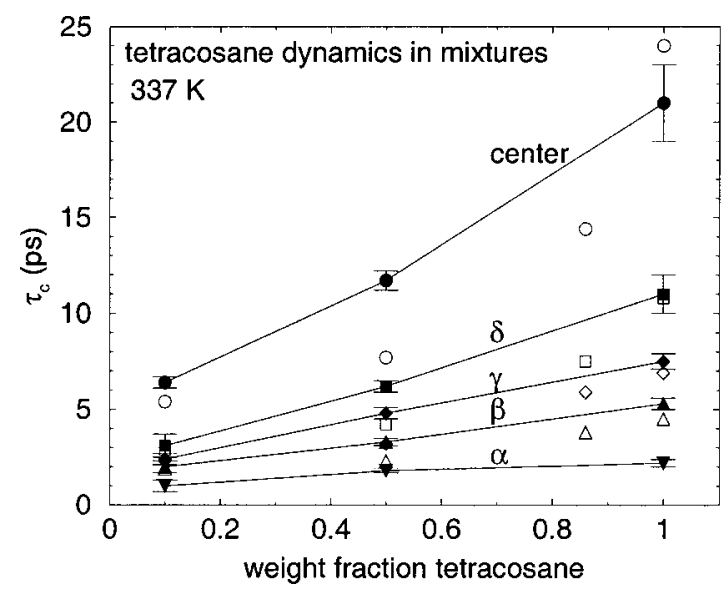

FIG. 9. Comparison of simulated and experimental correlation times for $\mathrm{C}-\mathrm{H}$ vectors of tetracosane in mixtures with hexane. Solid symbols are used for experimental data, and open symbols are used for simulation results using $15 \%$ higher torsion potential. 
TABLE VIII. Simulation results for 3 wt $\%$ hexane in tetracosane. ${ }^{a}$

\begin{tabular}{lccccccc}
\hline \hline \multicolumn{1}{c}{ Type } & $A$ & $\beta$ & $\begin{array}{c}\tau_{\mathrm{KWw}} \\
(\mathrm{ps})\end{array}$ & $\begin{array}{c}\tau_{0} \\
(\mathrm{ps})\end{array}$ & $\begin{array}{c}\tau_{c}(3 \%) \\
(\mathrm{ps})\end{array}$ & $\begin{array}{c}\tau_{c} \text { (neat) } \\
(\mathrm{ps})\end{array}$ & $\%$ change \\
\hline$\gamma$ hexane & 0.993 & 0.46 & 0.56 & 0.45 & 1.32 & 0.59 & -124 \\
$\beta$ tetracosane & 0.991 & 0.375 & 0.84 & 3.82 & 3.37 & 3.90 & 14 \\
$\gamma$ tetracosane & 0.94 & 0.354 & 1.13 & 2.22 & 5.27 & 6.1 & 14 \\
$\delta$ tetracosane & 0.89 & 0.343 & 1.62 & 1.98 & 8.00 & 8.4 & 4.8 \\
Center tetracosane & 0.78 & 0.296 & 2.55 & 3.78 & 20.4 & 20.0 & -2 \\
\hline \hline
\end{tabular}

${ }^{\mathrm{a}}$ Simulaltion performed with NVT dynamics $\left(T=316 \pm 4 \mathrm{~K} ; \rho=0.774 \mathrm{~g} / \mathrm{cc} ; P_{\mathrm{ave}}=-54 \mathrm{bar}\right)$.

Why can tetracosane results be made to match experiment with a minor modification to the torsion potential while hexane results cannot? We believe that this occurs because hexane, in contrast to long chain alkanes, rotates as a nearly rigid object. It has long been appreciated that local relaxation times in polymers are influenced both by conformational dynamics and overall molecular reorientation. ${ }^{34}$ The longer the molecule, the more conformational dynamics are the dominant influence. We believe from the evidence above that $\mathrm{C}-\mathrm{H}$ vector reorientation in hexane is almost completely determined by overall molecular reorientation and thus is controlled by interactions between sites on different molecules. This is precisely the aspect of the simulation which is treated least realistically in a UA model. In contrast, for long chain molecules, the effect of internal potentials (e.g., torsional barriers) on segmental dynamics is known to be very strong. Thus, for long chain molecules, any inadequacy in a UA model is less apparent.

We have also compared self-diffusion coefficients from the simulations of hexane to data in the literature. Interestingly, the self-diffusion coefficient of hexane calculated using the NERD force field is in good agreement with experimental results (Table IX). Included in that table, also, are results from other thermodynamically optimized UA and AUA force fields. The differences between force fields appear to be more of a function of temperature dependence rather than a superiority of any particular model. This comparison suggests that $T_{1}$ and NOE measurements provide a more stringent test of the ability of a force field to describe fast dynamic processes than do diffusion measurements.

\section{Mixtures}

Several mixtures were simulated at the experimental concentrations of $10 \mathrm{wt} \%, 50 \mathrm{wt} \%$, and $86 \mathrm{wt} \%$ tetracosane. The densities employed for these simulations were the ideal solution densities calculated from literature experimental values and equation of state interpolations. ${ }^{35,36}$
It is known that mixing chains of different lengths changes the dynamics of the chains from their behavior in pure fluids. Short chains are slowed by mixing with longer chains and the longer chains exhibit faster dynamics. Given the discrepancies between the simulated pure hexane results and experimental data, it is not surprising that the simulated correlation times for the mixtures are smaller than the experimental values (Fig. 9). Even for relatively little hexane in the mixture, the tetracosane correlation times are noticeably changed. A simulation with $3 \mathrm{wt} \%$ hexane in tetracosane (24 hexane chains, 197 tetracosane chains), for example, showed that the tetracosane correlation times decreased by up to $14 \%$ (Table VIII). The largest change is for the carbons closest to the ends, while the center carbons were unchanged. For hexane in this same mixture, the $\gamma$-carbon correlation time was increased by $124 \%$ compared with the pure fluid. A similar, noticeable effect on dynamic properties caused by the addition of a small amount of a second component has also been seen when a single decane molecule was added to a methane system. ${ }^{12}$ For the majority component, methane in that case, the viscosity, thermal conductivity, and diffusion were reduced by at least $20 \%$. These results illustrate the sensitivity of dynamic properties to impurities having different relaxation characteristics.

\section{CONCLUSION}

Multiple simulations for dynamic properties were performed with a united-atom force field that is known to reproduce thermodynamic properties quantitatively. A minor modification to the torsion potential gave simulated results for tetracosane which are in good agreement with experimental data for correlation times. For pure hexane and its mixtures with tetracosane, simulations exhibit deviations from experimental data. The simulated pure-hexane correlation times are about $45 \%$ shorter than experimental times; for the mixtures they are 20 to $60 \%$ shorter. Our results indicate that

TABLE IX. Self-diffusion coefficients of hexane.

\begin{tabular}{cccccc}
\hline \hline & & \multicolumn{3}{c}{ Self-diffusion coefficient $\left(10^{-9} \mathrm{~m}^{2} / \mathrm{s}\right)$} \\
\cline { 3 - 6 } Temperature (K) & Density (g/cc) & Expt. & NERD & TraPPE & AUA4 \\
\hline 273 & $0.677^{\mathrm{a}}$ & $2.96^{\mathrm{a}}$ & & & $3.6^{\mathrm{a}}$ \\
298 & $0.655^{\mathrm{b}}$ & $4.7^{\mathrm{b}}$ & 4.8 & $5.1^{\mathrm{b}}$ & $6.3^{\mathrm{a}}$ \\
333 & $0.622^{\mathrm{a}}$ & $5.97^{\mathrm{a}}$ & & & ${ }^{\mathrm{n}}$ \\
\hline \hline
\end{tabular}

${ }^{\mathrm{a}}$ Reference 3.

${ }^{\mathrm{b}}$ Reference 37. 
the UA model captures the main-chain relaxation processes of longer alkanes well; it is, however, insufficient to describe fast (subpicosecond), atomic-scale processes.

We conclude that, for simple alkanes of intermediate-tolarge molecular weight, a simple united-atom force field appears to be fully capable of describing thermodynamic and dynamic properties simultaneously with a reasonable level of confidence.

\section{ACKNOWLEDGMENTS}

This research was supported by the National Science Foundation through the Division of Material Research, Polymer Program (DMR-0099849) and through an instrument grant from NSF-CHE (9508244), and by the Division of Chemical Sciences, Office of Basic Energy Sciences, Office of Science, U.S. Department of Energy. Parts of this study were performed in the Instrument Center of the Department of Chemistry, University of Wisconsin-Madison, using instruments supported by NSF CHE-8813550, NSF CHE9629688, NSF CHE-9709065, NIH 1S10 RR04981-01. We thank XiaoHua Qiu for assistance with these experiments and for interpretation. We thank Shaun Lippow for data acquisition at $62.9 \mathrm{MHz}$.

\section{APPENDIX: MODEL FOR $J(\omega)$ FOR $\mathrm{C}_{24} \mathrm{H}_{50}$}

Based on our previous work on $\mathrm{C}_{44} \mathrm{H}_{90}$ and $\mathrm{C}_{153} \mathrm{H}_{208}$ melts, ${ }^{38}$ we expect the spectral density function for $\mathrm{C}_{24} \mathrm{H}_{50}$ to be reasonably well described by the following form:

$$
J(\omega)=A \tau_{1} /\left(1+\omega^{2} \tau_{1}^{2}\right)+(1-A) \tau_{2} /\left(1+\omega^{2} \tau_{2}^{2}\right) .
$$

Here $A$ is constrained to have the temperature dependence found in our previous work,

$$
A=0.915+73.957 / T-22688 / T^{2} .
$$

Fitting Eq. (A1) to data on $\mathrm{C}_{24} \mathrm{H}_{50}$ melts from 330 to 380 $K$, yields an excellent fit and the following parameters: $\tau_{1}$ $=6.7 \times 10^{-14} \exp (1900 / T) ; \tau_{2}=15.5 \tau_{1}$. We assume that this functional form is also a reasonable approximation for $\mathrm{C}_{24} \mathrm{H}_{50}$ in mixtures with hexane. From the above equations and parameters, we calculate $T_{1}^{\mathrm{DD}}(\omega) / T_{1}^{\mathrm{DD}}(0)$ versus $\operatorname{NOE}^{\mathrm{DD}}(\omega)$. Then we use this relationship and the observed value of $\mathrm{NOE}^{\mathrm{DD}}$, to find $T_{1}^{\mathrm{DD}}(0)$. Inserting this quantity into Eq. (8) yields our best estimate of $\tau_{c}$.

${ }^{1}$ S. K. Nath, F. A. Escobedo, and J. J. de Pablo, J. Chem. Phys. 108, 9905 (1998).

${ }^{2}$ M. G. Martin and J. I. Siepmann, J. Phys. Chem. B 102, 2569 (1998).

${ }^{3}$ P. Ungerer, C. Beauvais, J. Delhommelle, A. Boutin, B. Rousseau, and A. H. Fuchs, J. Chem. Phys. 112, 5499 (2000).
${ }^{4}$ G. D. Smith and D. Y. Yoon, J. Chem. Phys. 100, 649 (1994).

${ }^{5}$ P. V. K. Pant, J. Han, G. D. Smith, and R. H. Boyd, J. Chem. Phys. 99, 597 (1993).

${ }^{6}$ W. Paul, D. Y. Yoon, and G. D. Smith, J. Chem. Phys. 103, 1702 (1995).

${ }^{7}$ W. Paul, G. D. Smith, and D. Y. Yoon, Macromolecules 30, 7772 (1997).

${ }^{8}$ D. Y. Yoon, G. D. Smith, and T. Matsuda, J. Chem. Phys. 98, 10037 (1993).

${ }^{9}$ R. K. Bharadwaj and R. H. Boyd, Macromolecules 33, 5897 (2000).

${ }^{10}$ D. K. Dysthe, A. H. Fuchs, and B. Rousseau, Int. J. Thermophys. 19, 437 (1998).

${ }^{11}$ D. K. Dysthe, A. H. Fuchs, and B. Rousseau, J. Chem. Phys. 110, 4047 (1999).

${ }^{12}$ D. K. Dysthe, A. H. Fuchs, B. Rousseau, and M. Durandeau, J. Chem. Phys. 110, 4060 (1999).

${ }^{13}$ D. K. Dysthe, A. H. Fuchs, and B. Rousseau, J. Chem. Phys. 112, 7581 (2000).

${ }^{14}$ M. Mondello and G. S. Grest, J. Chem. Phys. 103, 7156 (1995).

${ }^{15}$ M. Mondello, G. S. Grest, A. R. Garcia, and B. G. Silbernagel, J. Chem. Phys. 105, 5208 (1996).

${ }^{16}$ M. Mondello and G. S. Grest, J. Chem. Phys. 106, 9327 (1997).

${ }^{17}$ M. Mondello, G. S. Grest, E. B. Webb III, and P. Peczak, J. Chem. Phys. 109, 798 (1998).

${ }^{18}$ S. K. Nath, F. A. Escobedo, J. J. de Pablo, and I. Patramai, Ind. Eng. Chem. Res. 37, 3195 (1998).

${ }^{19}$ M. L. Kaplan, F. A. Bovey, and H. N. Cheng, Anal. Chem. 47, 1703 (1975).

${ }^{20}$ N. Hedin and I. Furo', J. Magn. Reson. 131, 126 (1998).

${ }^{21}$ D. Gisser, S. Glowinkowski, and M. D. Ediger, Macromolecules 24, 4270 (1991).

${ }^{22}$ W. G. Hoover, Phys. Rev. A 31, 1695 (1985).

${ }^{23}$ S. Nosé, J. Chem. Phys. 81, 511 (1984).

${ }^{24}$ H. J. C. Berendsen, J. P. M. Postma, W. F. van Gunsteren, A. DiNola, and J. R. Haak, J. Chem. Phys. 81, 3684 (1984).

${ }^{25}$ D. B. Adolf, Ph.D. dissertation, University of Wisconsin-Madison, 1991.

${ }^{26}$ G. Williams and D. C. Watts, Trans. Faraday Soc. 66, 80 (1970); G. Williams, D. C. Watts, S. B. Dev, and A. North, ibid. 67, 1323 (1971); R. Kohlrausch, Prog. Ann. 12, 393 (1947).

${ }^{27}$ N. E. Moe and M. D. Ediger, Macromolecules 28, 2329 (1995).

${ }^{28}$ G. D. Smith, D. Y. Yoon, W. Zhu, and M. D. Ediger, Macromolecules 27, 5563 (1994).

${ }^{29}$ G. D. Smith, D. Y. Yoon, and R. L. Jaffe, Macromolecules 28, 5897 (1995).

${ }^{30}$ M. Kh. Karapet'yants and Y. Kuo-sen, Russ. J. Phys. Chem. 37, 1106 (1963).

${ }^{31}$ F. D. Rossini, K. S. Pitzer, R. L. Arnett, R. M. Braun, and G. C. Pimental, Selected Values of Physical and Thermodynamics Properties of Hydrocarbons and Related Compounds (Carnegie, Pittsburgh, PA, 1953).

${ }^{32}$ G. C. Chung, J. A. Kornfield, and S. D. Smith, Macromolecules 27, 964 (1994).

${ }^{33}$ G. D. Smith, W. Paul, M. Monkenbusch, L. Willner, D. Richter, X. H. Qiu, and M. D. Ediger, Macromolecules 32, 8857 (1999).

${ }^{34}$ D. R. Bauer, J. I. Brauman, and R. Pecora, Macromolecules 8, 443 (1975).

${ }^{35}$ TRC Thermodynamics Tables-Hydrocarbons, Thermodynamics Research Center, Texas A\&M University, College Station, TX.

${ }^{36}$ C. J. Peters, H. J. van der Kooi, and J. de Swaan Arons, J. Chem. Thermodyn. 19, 395 (1987).

${ }^{37}$ L. I. Kioupis and E. J. Maginn, Chem. Eng. J. 74, 129 (1999).

${ }^{38}$ X. H. Qiu and M. D. Ediger, Macromolecules 33, 490 (2000). 